\title{
Photon final states at the Tevatron
}

\author{
Mario Campanelli ${ }^{1}$ \\ Department of Physics and Astronomy \\ University College London UK
}

\begin{abstract}
We present here several recent measurements involving associate production of photons and jets at the Tevatron. In particular, inclusive photon + jet from D0, and photon + b-jets and photon + b-jet + leptons + MET from CDF are described in some detail. These measurements offer a good test of QCD predictions in rather complex final states.
\end{abstract}

\section{Introduction}

At first order in QCD perturbation theory, final state hard photons are produced directly from the hard scattering. For photon transverse momenta smaller than $120 \mathrm{Gev}$, the dominant production process is quark-gluon fusion, and the final-state photon can either be produced directly or from quark fragmentation. Since the quark PDF's are very well constrained by precision HERA data, the effect of measurements with photon final states will primarly be seen on gluon PDF's, or possibly on the heavy flavor ones. Another interest in the study of photon final states lies in the fact that they are predicted in all most popular extensions of the Standard Model.

\section{$2 \quad$ Experimental aspects}

The Tevatron, the most energetic particle accelerator in operation, started taking data for the Run II in 2001, and will continue until 2009 (discussions are under way to extend this date to 2010). At present, the machine is running very close to maximum performance, with its record luminosity of $3.12 \times 10^{32} \mathrm{~cm}^{-1} \mathrm{~s}^{-1}$, and has delivered $3.8 \mathrm{fb}^{-1}$.

Photon identification in the Tevatron environment suffers from the large neutral pion background. D0 has a Liquid Argon electromagnetic calorimeter with very good segmentation $(0.1 \times 0.1$ in $\Delta \eta \times \Delta \phi$, four times finer at the level of shower maximum), that allows distinction of photons and $\pi^{0}$ 's on a statistical basis using shower shape. The CDF calorimeter has a worse intrinsic granularity, but it is equipped with two wire chamber detectors, one just before the electromagnetic calorimeter, and the other at the location of the shower maximum; the comparison of the shower shape with distributions measured in test-beam allow also in this case a statistical discrimination of photons and pions.

If offline identification of a photon is difficult, even more is the case for photon-based triggers. Both experiments require isolated electromagnetic objects, without a matching track compatible with the cluster energy. In addition to the single photon trigger, CDF also uses a diphoton and tri-photon trigger, as well as a photon + muon, a photon + dijet and a photon + heavy flavor trigger. The efficiencies of these triggers are checked using $Z \rightarrow e e$ events.

\section{Photon + jet measurement from D0}

Using the inclusive photon trigger, the D0 collaboration has measured the cross section of production of photon + jets, as a function of photon $p_{T}$ for the four possible combinations

DIS 2008 
of the photon and the leading jet being in the forward or backward direction [1]. The ratio

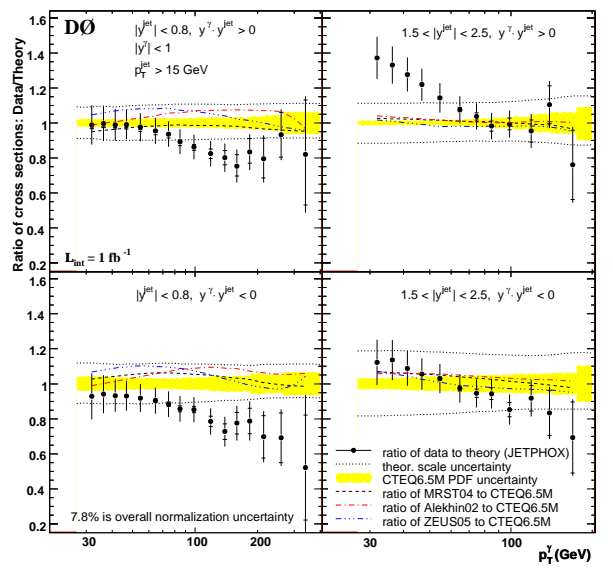

Figure 1: Ratio between observed cross section and NLO calculations (JatPhoX), for the four combinations of forward and backward photon and jet

of the observed cross section with respect to NLO predictions (JetPhoX [2]) are shown in figure 1. Some discrepancies are observed for same-side jets, as in previous measurements [3], probably due to an improper account of the interplay between fragmentation and soft terms.

\section{Photon + b-jet measurement from CDF}

The CDF experiment has installed in the beginning of Run II a device (SVT [4]) to measure track parameters online, and have this information available at trigger level. The possibility of selecting events with large impact parameter tracks allows the implementation of trigger paths enriched in heavy flavours. One example is the PHOTON_BJET dataset, that requires at trigger level the presence of a $20 \mathrm{GeV}$ jet that includes a track with impact parameter larger than $100 \mu$, and a photon with transverse momentum larger than $12 \mathrm{GeV}$. This is a large increse in phase-space with respect to the unbias photon trigger, whose threshold is at $25 \mathrm{GeV}$.

Since the trigger efficiency is difficult to simulate, depending on unstable beam conditions, it is directly measured from data using the control sample taken with the unbias photon trigger. Since the photon and the jet are separated, they are treated as independent objects. The efficiency of the SVT-based trigger is computed as the ratio of events passing it in the sample of those with a b-tagged jet and a photon above $25 \mathrm{GeV}$ (therefore passing the unbiased photon trigger). This efficiency has been found to be around $50 \%$, and independent on the photon $E_{t}$.

The analysis requires the jet to have an offline-reconstructed secondary vertex, and its axis to be separated from the photn by more than 0.7 in the $\Delta \phi-\Delta \eta$ plane. The b-purity of the secondary vertex tagged sample is extracted from data, fitting the invariant mass of the reconstructed secondary vertex. The result of the cross section measurement [5] is shown in figure 2, compared to the LO (Pythia [6]) predictions. The measured total cross section is 


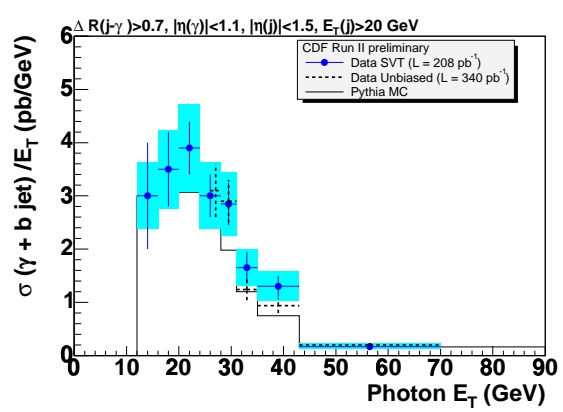

Figure 2: Cross section for events with a photon and a b-jet, as a function of the photon $E+T$

$90.5 \pm 6.0(\text { stat. })_{-15.4}^{+21.7} \mathrm{pb}$, to be compared to a Lo prediction of 69.3 . The main uncertainties are due to luminosity, and the uncertainties in tracking that determine the $\mathrm{b}$ purity.

\section{Photon + b-jets + lepton + MET signatures in CDF}

CDF also performed a signature-based search for new physics, looking for a photon in association with b-tagged jets, a lepton and missing ET [7]. The SM process that contributes to this final state in the case of two jets or more is $t \bar{t} \gamma$ production, a control sample for the $t \bar{t} H$ Higgs search channel at the LHC. The distribution of number of jets for data and the main background components is shown in figure 3, where the predomincance of the $t \bar{t} \gamma$ component for large jet multiplicities is visible.

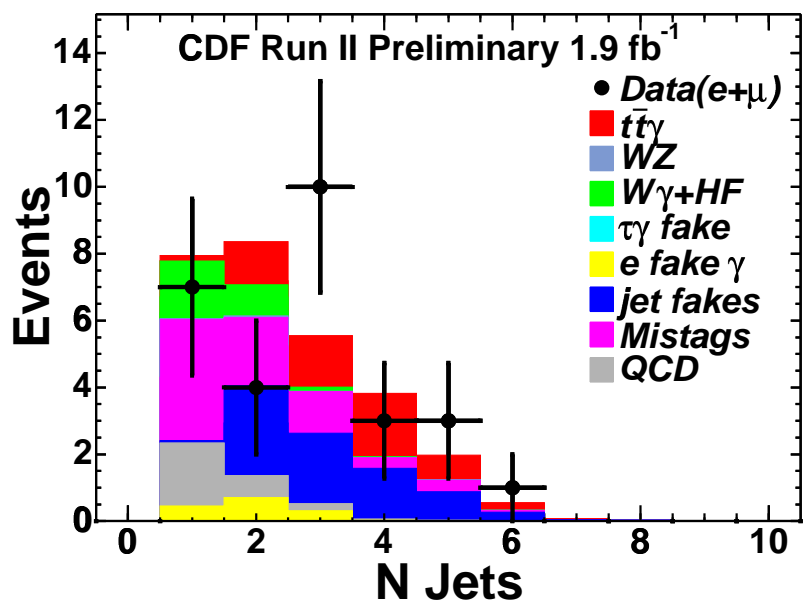

8

6

2

0 5

Figure 3: Distribution of number of jets for the CDF analysis of photon, lepton, MET and b-jet final states

The number of selected events with $1.9 \mathrm{fb}^{-1}$ (table 1) after the cut on the number of 


\begin{tabular}{|l|l|l|}
\hline$e$ & $\mu$ & $e+\mu$ \\
\hline $6.7 \pm 1.4$ & $4.4_{-0.8}^{+1.3}$ & $11.1_{-2.1}^{+2.3}$ \\
8 & 8 & 16 \\
\hline
\end{tabular}

Table 1: Number of $\mathrm{t} \bar{t} \gamma$ expected events for a luminosity of $1.9 \mathrm{fb}^{-1}$

jets is sufficient to claim the first observation of the $t \bar{t} \gamma$ final state, but the observed number of events is not in significant excess with respect to the predictions, so no new physics can be claimed.

\section{Conclusions}

Several measurements involving photon final states have been performed at the Tevatron, and here just some significant ones have been presented. They offer tests of QCD for a diversified set of final states; a slight discrepancy with theory predictions is confirmed in the $\gamma+$ jet channel, that requires some more work on the theory side to be better understood. For more complex final states, so far there is not enough precision to see discrepancies, but the road is paved to repeat these measurement with an improved statistics.

\section{References}

[1] arXiv:/0804.1107 [hep-ex] submitted to Phys. Lett. B

[2] P. Aurenche, J.Linfords, Nucl. Phys. B168, 296 (1980), P/ Aurenche et al. Phys. Lett. B 140, 87 (1984); Zeit. Phys.C 29, 423 (1985)

[3] J. Alitti et al. (UA2 Coll.), Phys. Lett B 263, 544 (1991); D.Acosta et al. (CDF Coll.), Phys. Rev. D 65, 112003 (2002); V.Abazov et al.(D0 Coll.), Phys. Lett. B 659, 151 (2006)

[4] W.Ashmanskas et al. Nucl. Instrum. Meth., A 447218 (2000)

[5] Public note is at http://www-cdf.fnal.gov/physics/new/qcd/photon_b_SVT_06/cdf8623_pub_bgamma.ps

[6] T.Sjostrand et al., Comp. Phys. Comm. 135, 238 (2001)

[7] Public note is at http://www-cdf.fnal.gov/physics/exotic/r2a/20080515.ttbargamma/ttg_public_2fb/ttgamma_public.pdf 